\title{
Results from the first missions of the JEM-EUSO program
}

\author{
Mario Bertaina ${ }^{1,2 *}$ On behalf of the JEM-EUSO Collaboration \\ ${ }^{1}$ Department of Physics, University of Turin - Italy \\ ${ }^{2}$ National Institute for Nuclear Physics (INFN) - Turin, Turin - Italy
}

\begin{abstract}
The origin and nature of Ultra-High Energy Cosmic Rays (UHECRs) remain unsolved in contemporary astroparticle physics. To give an answer to these questions is rather challenging because of the extremely low flux of a few per $\mathrm{km}^{2}$ per century at extreme energies such as $\mathrm{E}>5 \times 10^{19} \mathrm{eV}$. The objective of the JEMEUSO program, Extreme Universe Space Observatory, is the realization of a space mission devoted to scientific research of cosmic rays of highest energies. Its super-wide-field telescope will look down from space onto the night sky to detect UV photons emitted from air showers generated by UHECRs in the atmosphere. The JEM-EUSO program includes different missions using fluorescence detectors to make a proof-of-principle of the UHECR observation from space and to raise the technological level of the instrumentation to be employed in a space mission. EUSO-TA, installed at the Telescope Array site in Utah in 2013, is in operation. It has already detected 9 UHECRs in coincidence with Telescope Array fluorescence detector at Black Rock Mesa. EUSO-Balloon flew on board a stratospheric balloon in August 2014. It measured the UV intensity on forests, lakes and the city of Timmins as well as proved the observation of UHECR-like events by shooting laser tracks. EUSO-SPB was launched on board a super pressure balloon on April $25^{\text {th }}$ and flew for 12 days. It proved the functionality of all the subsystems of the telescope on a typical duration of a balloon flight; observed the UV emission on oceans and has a self-trigger system to observe UHECRs with energy $\mathrm{E}>3 \times 10^{18} \mathrm{eV}$. TUS, the Russian mission on board the Lomonosov satellite in orbit since April $28^{\text {th }} 2016$, is now included in the JEMEUSO program and has detected so far in the UHECR trigger-mode a few interesting signals. Mini-EUSO is in its final phase of integration in Italy, where several performance tests are being held. Mini-EUSO will be installed inside the International Space Station (ISS) in 2019. The main results obtained so far by such missions are summarized and put in prospect of future space detectors such as K-EUSO and POEMMA.
\end{abstract}

\section{Introduction}

The origin and nature of Ultra-High Energy Cosmic Rays (UHECRs) remain unsolved in contemporary astroparticle physics [1]. To give an answer to these questions is rather challenging because of the extremely low flux of a few per $\mathrm{km}^{2}$ per century at extreme energies such as $E>5 \times 10^{19} \mathrm{eV}$ (EECRs). The objective of the JEMEUSO program, Extreme Universe Space Observatory, is the realization of a space mission devoted to scientific research of EECRs [2]. Its super-wide-field telescope will look down from space onto the night sky to detect UV photons emitted from Extensive Air Showers (EAS) generated by EECRs in the atmosphere.

The JEM-EUSO program includes several missions from ground (EUSO-TA [3]), from stratospheric balloons (EUSO-Balloon [4], EUSO-SPB [5, 6]), and from space (TUS [7], Mini-EUSO [8]) employing fluorescence detectors to make a proof-of-principle of the EECR observation from space and to raise the technological level of the instrumentation to be employed in space missions such as K-EUSO [9] and POEMMA [10] . A general description of the JEM-EUSO program and its scientific objectives is reported in [11]. A space project devoted to the study of

*e-mail: bertaina@to.infn.it

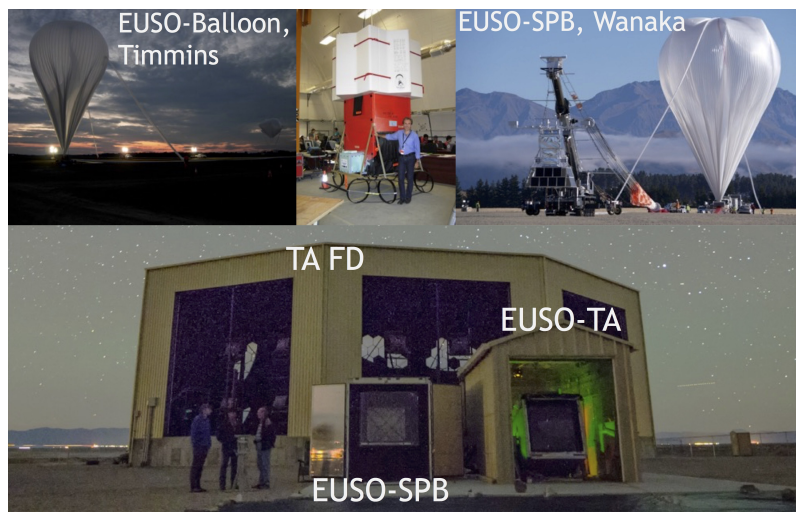

Figure 1. EUSO-TA and stratospheric balloon missions of the JEM-EUSO program. See text for details.

EECRs such as JEM-EUSO should have enough quality in terms of exposure determination and EAS parameter reconstruction to satisfy the scientific requirements of such an ambitious mission. A review of the key results obtained by each experiment of the JEM-EUSO program in this respect is described in the following (details can be found in [3] - [22]). 


\section{EUSO-TA}

EUSO-TA is a ground-based telescope, installed at the TA site in Black Rock Mesa, Utah, USA. This is the first detector to successfully use a Fresnel lens based optical system and Multi-Anode Photomultipliers (MAPMT, 64 channels per tube, 2304 channels encompassing a $10.6^{\circ} \times$ $10.6^{\circ}$ field of view - FoV) for detection of UHECRs. The telescope is located in front of one of the fluorescence detectors of the TA experiment (see Fig. 1). Since its operation in 2015, the detector has observed several UHECRs and, in addition, meteors among other atmospheric phenomena such as lightning. The detection of meteors is important in view of the search for particular types of Strange Quark Matter such as nuclearites which will be performed with the upgraded version of the detector which is going to start taking data in 2019. By summing up consecutive frames $(\sim 3 \mathrm{~ms})$, it was possible to reach the limiting magnitude of 5.5 to the detection of these kind of slow events. Measurements of the UV night sky emission in different conditions and moon phases have been performed. These observations serve as a proof of concept for the future application of this detector technology for space-based missions. The key results of EUSO-TA in view of the measurements of UHECRs from space is reported in the following. A detailed review of EUSO-TA results and its prospects can be found in [12].

Fig. 2 shows an example of a UHECR which has been detected by EUSO-TA, using TA external trigger. It can be seen that re-binning of the images significantly increases the visibility of the tracks - making EUSO-TA data more similar to those of ground-based UHECR telescopes, which have much larger pixel sizes. However, such a pixel size is not suited for space-based observations, to which EUSO-TA has been tuned. Simulations of the events made with the OffLine package [13] are also presented. The shower image can be reproduced to very fine detail, taking into account the uncertainties in EAS reconstruction by the Fluorescence Detector of Telescope Array (TAFD) and intrinsic modeling of the detector response. With the implementation of the external trigger, data is collected for each TAFD event. Therefore, an event is considered as detected if a linear trace is found in the EUSO-TA data and a corresponding event in TAFD results. To date, 9 UHECR events (see Fig. 3) have been identified in 130 hours of UHECRdedicated observations. The distances of these events from the detector vary between approximately 1 and $9 \mathrm{~km}$, while the energy is between $10^{17.7}-10^{18.8} \mathrm{eV}$, according to the TAFD reconstruction. In 2016, simulations were performed, using the updated detector parameters and ESAF (EUSO Simulation and Analysis Framework) simulation code [13], resulting in 8 predicted events, consistent with the 9 UHECRs observed to date, even though some independent assumptions had to be made on EUSO-TA intrinsic sensitivity to UHECRs. Nevertheless, this result confirms the general understanding of the detector response through simulations. The proximity of the events and the dead time between frames makes events visible in the detector for a duration of 1 or 2 frames. EUSO-TA does not usually observe the EAS maximum, but a late stage

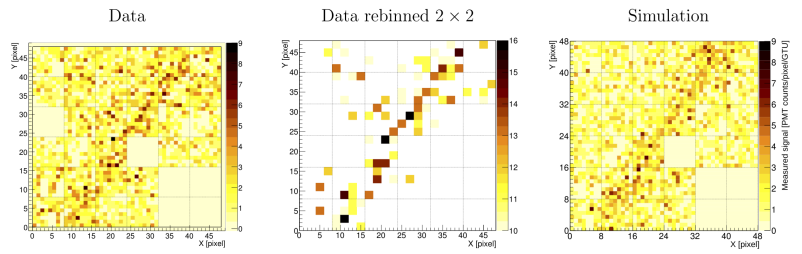

Figure 2. Example of a UHECR observed by EUSO-TA (E $\sim 10^{18} \mathrm{eV}$, impact parameter $2.5 \mathrm{~km}$ with respect to the telescope, zenith angle of the axis of $35^{\circ}$ and azimuth angle of $7^{\circ}$ ) with different pixel binning and comparison with simulations. The left plot shows the real data in photoelectron counts, in the centre $2 \times 2$ rebinning of the data is shown and in the right plot the simulation made with the OffLine package [13].

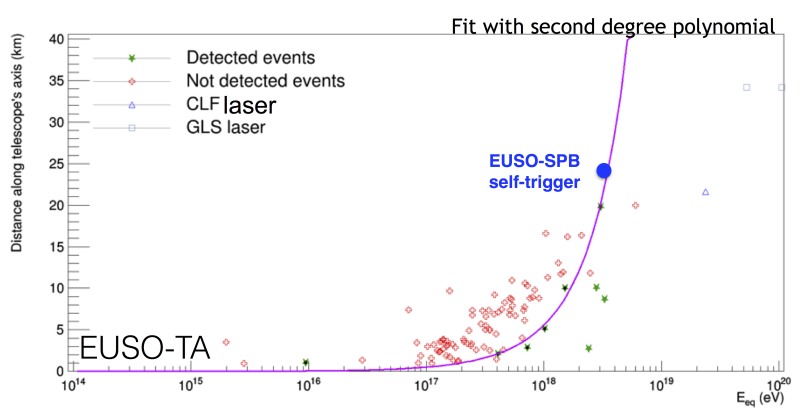

Figure 3. All UHECRs detected by TAFD in the EUSO-TA FoV during its operation with non-detected events and laser shots superimposed. The vertical axis shows the distance to the shower axis. The horizontal axis shows the events equivalent energy. The fit to the detected points suggests an estimate of EUSO-TA detection energy threshold. The blue point indicates the estimated energy threshold of EUSO-SPB determined during the field tests.

of the shower development, and as such the number of registered photons corresponds to an EAS of lower energy than if the instrument was optimally pointed towards the shower maximum. Therefore, to estimate the instrument's capabilities it was necessary to calculate the equivalent energies of the events $\left(E_{e q}\right)$, corresponding to the reconstructed energy assuming that EUSO-TA observed the event's shower maximum. This calculation is based on the parameters measured by TA for each individual shower. The corresponding points can be used to form a conservative estimate of the detector's energy threshold. The empirical relationship that describes the energy threshold $\left(E_{e q}\right)$ at different distances from the shower axis $\left(R_{p}\right)$ is a second degree polynomial function. It can be seen that the strong signals of the TA Central Laser Facility (CLF) shots (EAS equivalent energy of $\sim 10^{19.4} \mathrm{eV}$ at a distance of $21 \mathrm{~km}$ ) and Ground Laser System (GLS) shots (EAS equivalent energy of $\sim 10^{19.7} \mathrm{eV}$ and $\sim 10^{20} \mathrm{eV}$ at a distance of $33 \mathrm{~km}$ ) are on the right side of the curve, i.e. in the detectable region, as expected. Moreover, the groundfield tests performed with EUSO-SPB employing an internal trigger logic, indicate that its energy threshold lies on top of the fitting curve supporting the conclusions derived with such method (see Fig. 3), though EUSO-SPB over- 
all detector efficiency is $\sim 2$ times better than EUSO-TA (see details in section 4). In other words the energy shift between the sensitivity to an UHECR, recognized by the external trigger of a more performing detector, and the autonomous capability of trigger UHECR events is a factor $\sim 2$.

\section{EUSO-Balloon}

EUSO-Balloon [4] was launched by CNES from the Timmins base in Ontario (Canada) on the moonless night of August 25, 2014 UT. After reaching the floating altitude of $\sim 38 \mathrm{~km}$, EUSO-Balloon imaged the UV intensity in the wavelength range $290-430 \mathrm{~nm}$ for more than 5 hours before descending to ground. The refractor telescope consisted of a similar apparatus as EUSO-TA (two Fresnel lenses of $\sim 1 \mathrm{~m}^{2}$ size and a Focal Surface (FS) filled with MAPMTs). The spatial and temporal (GTU) resolutions of the detector were $130 \mathrm{~m}$ and $2.5 \mu \mathrm{s}$, respectively. The full $\mathrm{FoV}$ in nadir mode was $\sim 11^{\circ}$. During 2.5 hours of EUSO-Balloon flight, a helicopter circled under the balloon operating UV flashers and a UV laser to simulate the optical signals from UHECRs, to calibrate the apparatus, and to characterise the optical atmospheric conditions. During flight EUSO-Balloon took more than 2.5 million images that have been analysed to infer different information: study of the performance of the different parts of the detector; response of the detector to the UV flasher and laser events; UV radiance from the Earth atmosphere and ground in different conditions: clear and cloudy atmosphere, forests, lakes, as well as city lights.

The measurement of UV light intensity is relevant for a JEM-EUSO-like mission as it is one of the key parameters to estimate the exposure curve as a function of energy [14]. However, EUSO-Balloon uses a very different approach compared to previous measurements as it is based on an wide-FoV and wide-bandwidth optical refractive system with very fine spatial and temporal resolutions, which requires a careful computation of the optics and detector response to translate the detected counts into an absolute measurement. From the point of view of the capability of a space-based observatory for EECRs the essential point is the number of counts per GTU at pixel level which is the pedestal that should be dark enough to detect a EECR track on top. Fig. 4 shows the average normalized count rates $\langle\hat{N}\rangle$ as a function of the packet time (breaks are due to technological tests foreseen for this flight). The count rate, $\hat{N}_{0}$, for clear atmosphere conditions is an input parameter to the EAS simulations used to estimate the reference aperture for EECR observations. The corresponding absolute intensity $\left(I_{0}\right)$ is useful for the design of any spacebased EECR observatory, independent of the configuration of the instrument. In EUSO-Balloon, only the backscattered light from the airglow and extraterrestrial light contributes to the measured diffuse light. The reflectivity of the clouds is expected to be higher than clear atmospheric conditions. Thus, the interval and area with lowest count rates is assumed to represent clear atmosphere. Such conditions were assumed to be present between 04:38 and 04:52. Based on the average of the distribution in that time

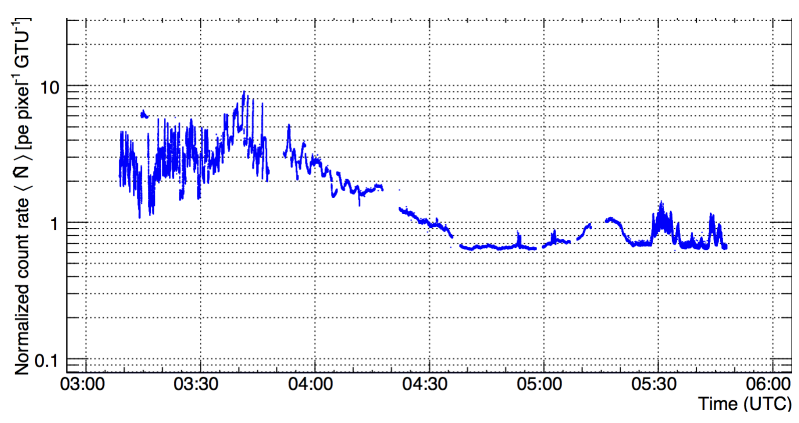

Figure 4. Average normalized count rates $\langle\hat{N}\rangle$ as a function of the packet time [15].
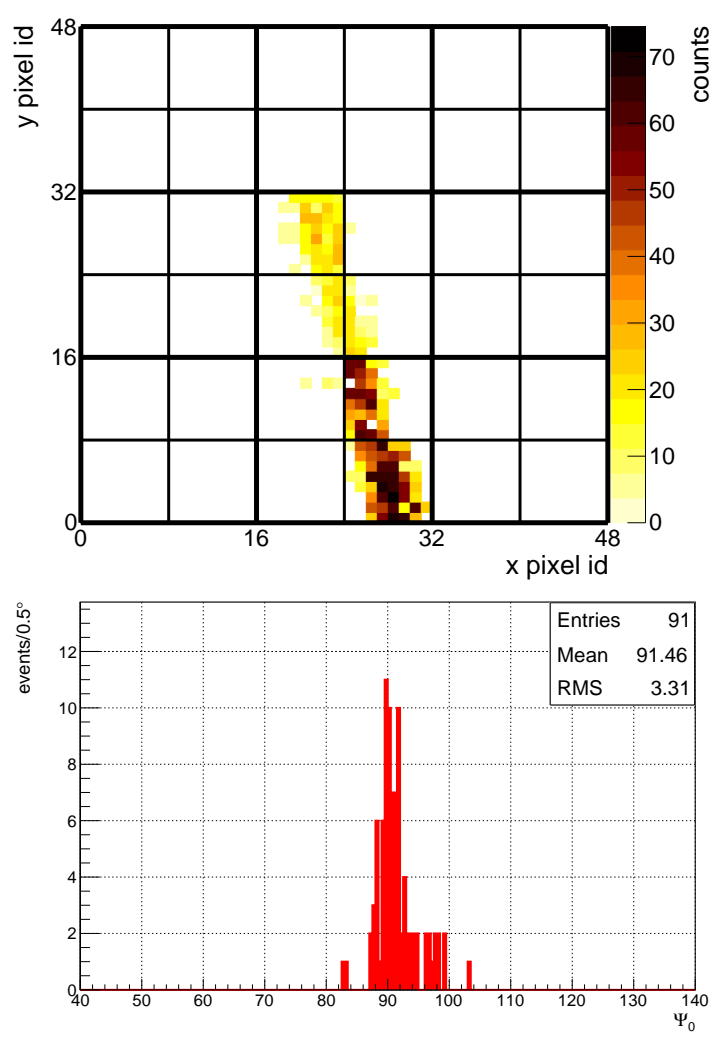

Figure 5. Left: Example of a laser track detected by EUSOBalloon. Right: Zenith angle reconstruction of the helicopter laser shots with the 2-parameter fit method and laser energy of $15 \mathrm{~mJ}[16]$.

window, the reference $\hat{N}_{0}$ value is $\sim 0.65$, the FWHM of the pixel distribution being $\sim 0.03$. Between 04:20 and the end of measurement, when the artificial lights of Timmins and surroundings were out of the FoV, the count rate varies within a factor of $\sim 2$. This gives the maximal difference of UV intensity between clear and cloudy sky conditions during flight. Ray trace simulations were then performed using the OffLine code to translate $\hat{N}_{0}$ in $I_{0}$ values. In the area with no artificial light sources, based on the airglow and starlight models, the measured count rate from the diffuse light under clear atmosphere conditions corresponds to $I_{0}=300-320$ photons $\mathrm{m}^{-2} \mathrm{sr}^{-1} \mathrm{~ns}^{-1}$ in the $300-500 \mathrm{~nm}$ 


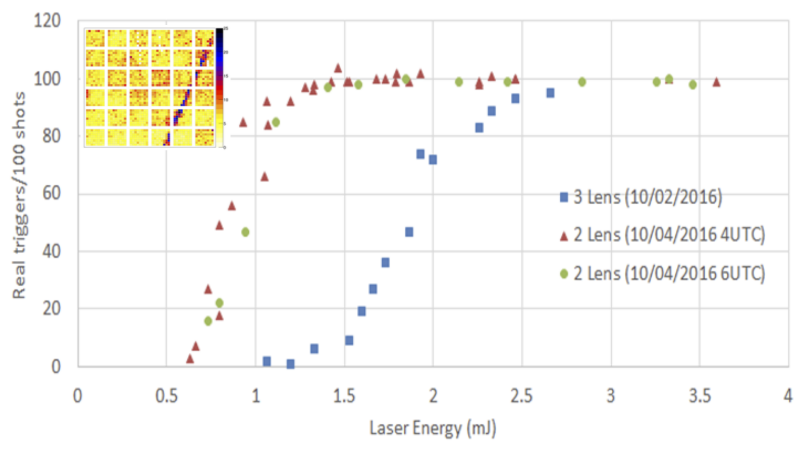

Figure 6. EUSO-SPB trigger efficiency as a function of GLS energy with 2 and 3 lens system for vertical shots during the field tests in Utah. An example of GLS track ( $2 \mathrm{~mJ}$ energy) is displayed on the top-left corner.

band. This value is in the band of previous measurements and confirms a good understanding of the detector performance also in this respect, which is very important in view of JEM-EUSO. A detailed description of the results related to the UV nightglow measurements during EUSO-Balloon flight can be found in [15].

The helicopter events revealed to be extremely useful to understand the system's performance and to test the capability of EUSO-Balloon to detect and reconstruct EASlike events. Laser tracks are used to test the reconstruction algorithms [16]. The analysis is based on the geometry of the triggered pixels. The typical time fit of a laser event and the direction reconstruction are shown in Fig. 5 after requiring basic quality cuts: a) a set of events of same energy ( $15 \mathrm{~mJ}$, equivalent to $\sim 10^{20} \mathrm{eV}$ EAS); b) a track lasting at least 4 GTUs. It is important to remember that the read-out period of $2.5 \mu$ is optimised for JEM-EUSO, which is expected to detect EAS at $\sim 400 \mathrm{~km}$ distance, instead of $\sim 35 \mathrm{~km}$ as in case of EUSO-Balloon.

\section{EUSO-SPB}

EUSO-SPB1 was launched April 252017 from Wanaka New Zealand as a mission of opportunity on a NASA SPB (Super Pressure Balloon) test flight planned to circle the southern hemisphere [5]. The primary scientific goal was to make the first observation of UHECR-EASs by looking down on the atmosphere with an optical fluorescence detector from the near space altitude of $33 \mathrm{~km}$. After 12 days 4 hours aloft, the flight was terminated prematurely in the Pacific Ocean about $300 \mathrm{~km}$ SE of Easter Island due to a leak in the balloon. The telescope was similar to EUSO-Balloon. An autonomous internal trigger was implemented according to [17] to detect UHECRs. About 30 hours of useful data have been collected during night time.

In October 2016, the fully assembled EUSO-SPB detector was tested for a week at the EUSO-TA site to measure its response and to calibrate it by means of a portable GLS. Observations of CLF, stars, meteors were performed.

Fig. 6 shows an example of a GLS track detected by EUSO-SPB as well as the trigger efficiency curve of the 2 and 3 lens system as a function of GLS energy. With the

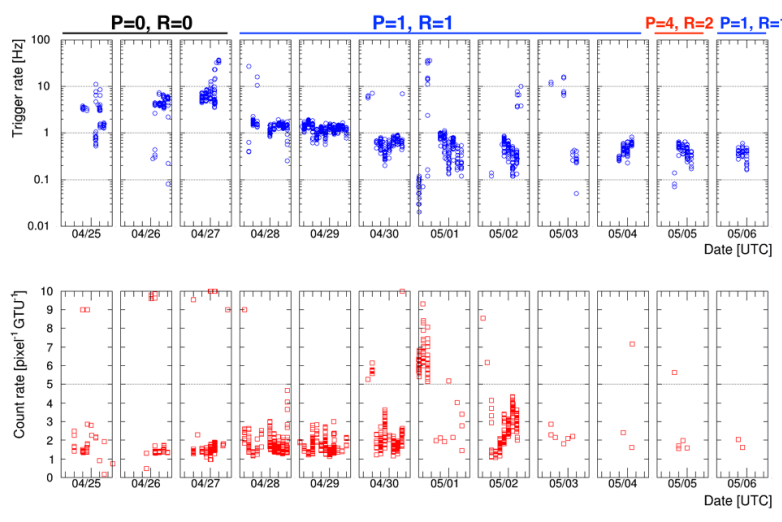

Figure 7. Trigger rate (top) and average count rate measured at pixel level (bottom) measured by EUSO-SPB in flight.

2 lens system, which is the one that flew from Wanaka, the $\sim 50 \%$ trigger efficiency is reached at laser energies whose luminosity is equivalent to $\sim 45^{\circ}$ inclined EAS of $\mathrm{E} \sim 3 \times 10^{18} \mathrm{eV}$ seen from above by a balloon flying at 33 $\mathrm{km}$ altitude. This condition is represented by the blue point displayed in Fig. 3. Fig. 7 shows the average pixel count and trigger rates during flight. The pixel count rate will be used to determine the exposure and the UV intensity as performed for EUSO-Balloon. The different intensity levels are due to clear atmosphere, clouds and presence of moon light. The trigger rate was typically a few $\mathrm{Hz}$, which is compliant with JEM-EUSO requirements. A more detailed description of the trigger performance on flight and in Utah can be found in [18]. The analysis of the collected data is on going. Tracks of CRs directly crossing the detector have been recognized. However, no EAS track has been clearly identified yet. Simulations post-flight indicate that the number of expected events is $\sim 1$ in the available data sample independently of the balloon height and UV level confirming pre-flight expectations for such a flight duration. Analyses are on going to take properly into account the role of clouds and search for candidates. A detailed description of the analysis on the role of clouds and on the estimation of the exposure is reported in [19].

As mentioned before a more ambitious mission is currently under development, EUSO-SPB2 [6]. It will be equipped with 2 telescopes. One telescope will be devoted to UHECR measurements using the fluorescence technique. The FS will be equipped with 3 PDMs to increase the UHECR collection power. A more performing optics (Schmidt camera) and a reduced GTU $(1 \mu \mathrm{s})$ will lower the energy threshold of the instrument. The FS of the other telescope will be based on SiPMT sensors and a dedicated electronics to detect the Cherenkov emission in air by UHECR-generated EASs. In perspective it will test the capability to detect EAS generated by $v_{\tau}$ interacting in the Earth crust. For this observation the detector will be pointing slightly below the limb. EUSO-SPB2 is expected to fly by 2022 from Wanaka, New Zealand. 


\section{TUS \& Mini-EUSO}

The Track Ultraviolet Setup (TUS) detector was launched on April 28, 2016 as a part of the scientific payload of the Lomonosov satellite. TUS [7] is the world's first orbital detector aiming at detecting EECRs. The satellite has a sun-synchronous orbit with an inclination of $97.3^{\circ}$, a period of $\sim 94 \mathrm{~min}$, and a height of $470-500 \mathrm{~km}$. The telescope consists of two main parts: a modular Fresnel mirror-concentrator with an area of $\sim 2 \times 2 \mathrm{~m}^{2}$ and 256 PMTs arranged in a $16 \times 16$ photo-receiver matrix located in the focal plane of the mirror. The pixel's FoV is 10 $\mathrm{mrad}$, which corresponds to a spatial spot of $\sim 5 \mathrm{~km} \times 5$ $\mathrm{km}$ at the sea level from a $500 \mathrm{~km}$ orbit height. Thus, the full area observed by TUS at any moment is $\sim 80 \mathrm{~km} \times$ $80 \mathrm{~km}$. TUS is sensitive to the near UV band with a time resolution of $0.8 \mu \mathrm{s}$ in a full temporal interval of 256 time steps. During its operation TUS has detected about $8 \times 10^{4}$ events that have been subject to an offline analysis to select among them those satisfying basic temporal and spatial criteria of EECRs. A few events passed this first screening. To perform a deeper analysis of such candidates, a dedicated version of ESAF as well as a detailed modeling of TUS optics and detector are being developed [21]. One event is right now subject to deep analysis. A detailed study of this event is presented elsewhere [20] which includes also the study of weather conditions and presence of artificial lights. This example shows the importance of TUS measurements to determine analysis strategies in view of K-EUSO.

Mini-EUSO [8] is a UV telescope to be placed in 2019 inside the ISS, looking down on the Earth from a nadirfacing window in the Russian Zvezda module. MiniEUSO will map the earth in the UV range (290 - 430 $\mathrm{nm}$ ) with a spatial and temporal resolutions of $\sim 5 \mathrm{~km}$ (like TUS) and $2.5 \mu \mathrm{s}$, respectively. Mini-EUSO has a FS similar to EUSO-TA. The optical system consists of 2 Fresnel lenses of $25 \mathrm{~cm}$ diameter with a large $\mathrm{FoV}$ of $\sim 19^{\circ}$. A multiple level trigger [22] will allow the measurement of UV transients at different time scales, complementing TUS observations. Laboratory experiments with Mini-EUSO engineering model and simulations confirm the sensitivity of Mini-EUSO to EECR-like transients around $10^{21} \mathrm{eV}$. The Engineering Model of Mini-EUSO has been tested successfully in laboratory (TurLab facility) and in night observations to check the trigger performance, the response to anthropogenic lights such as airplanes, city lights and satellites reflecting sun light during astronomical twilight, as well as to natural lights from stars, UV nightglow and meteors to determine its sensitivity.

\section{Conclusions}

Different missions from ground, stratospheric balloon and from space have been developed within the JEM-EUSO program to address relevant key aspects in view of a main mission, such as K-EUSO and POEMMA: a) raise the Technological Readiness Level (TRL) of the different components of the JEM-EUSO detector; b) prove the capability of the JEM-EUSO approach to detect UHECRs from space; c) verify the quality of the reconstruction of EAS parameters; d) define methodologies to evaluate the instantaneous exposure of the instrument in different atmospheric and observational conditions. The current TRL of the electronics and optics is around 7. The flight of Mini-EUSO will further increase the TRL of the system.

The capability of the JEM-EUSO approach to detect UHECR from space was checked by means of EUSOTA and EUSO-SPB in Utah, as well as EUSO-Balloon in space. EUSO-TA detected 9 UHECRs in coincidence with TA in the energy range $10^{17.7}-10^{18.8} \mathrm{eV}$. This result is consistent with simulations, indicating the quality of the simulation software to provide reliable expectations also for the space observations of UHECRs. It is important to underline that EUSO-TA detected EAS at a few $\mathrm{km}$ distance from the detector, while the instrument was fine tuned to detect events at several hundreds $\mathrm{km}$ distance. EUSO-TA detected also artificial events designed to mock up EAS cascades generated by means of laser shots at different distances from the detector (between $20 \mathrm{~km}$ and 100 $\mathrm{km})$. While EUSO-TA detected real EASs in a passive way, through a trigger induced by the Telescope Array experiment, the EUSO-SPB calibration campaign in Utah allowed to test the same trigger logic developed for JEMEUSO. In this case, artificial tracks were used by means of a mobile laser system and the central laser facility of TA. Simulations successfully reproduced the laser intensity of events within $20 \%$ systematic difference. Extrapolations indicated that EUSO-SPB flying on a stratospheric balloon would have been sensitive to EAS of energy $\mathrm{E}>3$ $\times 10^{18} \mathrm{eV}$. By taking into account the different size of the instruments, the background light intensity and the EAS distance, these results translate into an energy threshold for JEM-EUSO of $\mathrm{E} \sim 5 \times 10^{19} \mathrm{eV}(\sim 50 \%$ trigger efficiency). Such value is only $\sim 50 \%$ higher than expectations from simulations. The difference can be easily bridged by the EUSO-SPB2 which will employ higher performing electronics and optics. And it is important to remind here that the balloon observation still suffers from the relatively close distance of EAS, for which the JEM-EUSO instrumentation is not optimized. This is an important result and provides a solid base for the expected performance of a space-based mission like K-EUSO or POEMMA.

While EUSO-TA and EUSO-SPB proved the possibility of observation from the ground, EUSO-Balloon recorded laser tracks in flight. Such artificial EAS-tracks were shot from a helicopter circling underneath the balloon during the flight. A total of 275 events with an equivalent energy of $5-10 \times 10^{19} \mathrm{eV}$ were detected, well compatible with an expected number of $261 \pm 16$. Finally, the TUS telescope, using a technology developed for ground experiments, detected several events in the UHECR trigger mode. A simple selection algorithm shows that a few of them have characteristics similar to EAS events. A detailed analysis of these events and comparison with simulations is ongoing to establish their origin. This takes into account similarities in the time development of the light intensity of the signal, its speed, the equivalent EAS energy, the location of the events and the weather conditions at the place and time at which they occurred. 
The quality of the direction reconstruction of the events was performed using EUSO-TA and EUSO-SPB detected laser tracks in Utah as well as the helicopterbased laser events for EUSO-Balloon. The angular reconstruction of EUSO-TA and EUSO-SPB detected laser tracks have an uncertainty of $\sim 0.5 \mathrm{deg}$. This is much better than the requirements for JEM-EUSO $\left(\leq 2.5 \mathrm{deg}\right.$ for $10^{20}$ eV EAS). The advantage here is due to the knowledge of the position of the light source. In the case of EUSOBalloon the quality decreased to $\sim 3.1 \mathrm{deg}$. This result is however promising for JEM-EUSO, because EUSOBalloon was expected to show a poorer angular resolution, due to the close distance of the EAS and the relatively long time-bin integration, which doesn't match the high-speed movement of the track through the detector.

EUSO-Balloon and EUSO-SPB flight measurements, as well as the data collected by TUS, revealed to be extremely useful to define methodologies to evaluate the instantaneous exposure of the instrument in different atmospheric and observational conditions. The EUSO-Balloon flight proved to be autonomously capable to recognize the presence of anthropogenic fixed and pulsed lights all along the trajectory. In some cases, lights were found in places not recorded in NASA satellite data. In the case of EUSO-SPB, to determine the exposure all along the flight, Weather Research and Forecasting model is being used to establish the presence of clouds, their optical depth and the resulting limitations of the nominal exposure of the instrument. Similar methodology is being applied to TUS data for the search of UHECRs and nuclearites. Such results are being cross-checked and validated with satellite data and appear to be promising. In this context, Mini-EUSO will be extremely useful, because it will allow to complement the observations of TUS using a more performant electronics, a wider coverage area, and an instrumentation similar to that of K-EUSO and POEMMA.

\section{Acknowledgments}

This work was partially supported by Basic Science Interdisciplinary Research Projects of RIKEN and JSPS KAKENHI Grant (22340063, 23340081, and 24244042), by the Italian Ministry of Foreign Affairs and International Cooperation, by the Italian Space Agency through the ASI INFN agreement n. 2017-8-H.0, by contract contract 2016-1-U.0, by NASA award 11-APRA-0058 in the USA, by the Deutsches Zentrum für Luft- und Raumfahrt, by the French space agency CNES, the Helmholtz Alliance for Astroparticle Physics funded by the Initiative and Networking Fund of the Helmholtz Association (Germany), and by Slovak Academy of Sciences MVTS JEMEUSO as well as VEGA grant agency project 2/0132/17. Russia is supported by ROSCOSMOS and the Russian Foundation for Basic Research Grant No 16-29-13065, and the Olle Engkvist Byggmästare Foundation.

\section{References}

[1] B.R. Dawson, M. Fukushima and P. Sokolski P., Prog. Theor. Exp. Phys. 12 12A107 (2017).

[2] J.H. Adams et al (JEM-EUSO Coll.), Experimental Astronomy 403 (2015).

[3] G. Abdellaoui et al (JEM-EUSO Coll.), Astroparticle Physics 10298 (2018).

[4] J.H. Adams et al (JEM-EUSO Coll.), Experimental Astronomy 40281 (2015).

[5] L. Wiencke and A. Olinto for the JEM-EUSO Coll., PoS(ICRC2017), 1097 (2017).

[6] J.H. Adams et al, ArXiv e-prints [[arXiv]1703.04513 (2017).

[7] P. Klimov et al (TUS Coll.), Space Science Reviews 8 1 (2017).

[8] F. Capel et al, Advances in Space Research 10.1016/j.asr.2017.08.030 (2017).

[9] M. Casolino M. et al (JEM-EUSO Coll.), PoS(ICRC2017) 368 (2017).

[10] A. Olinto et al (POEMMA Coll.), PoS(ICRC2017) 542 (2017).

[11] E. Parizot et al (JEM-EUSO Coll.), This Conference Proceedings (2018).

[12] F. Bisconti et al (JEM-EUSO Coll.), This Conference Proceedings (2018).

[13] F. Bisconti F. et al (JEM-EUSO Coll.), PoS(ICRC2017) 463 (2017).

[14] J.H. Adams et al (JEM-EUSO Coll.), Astroparticle Physics 4476 (2013).

[15] G. Abdellaoui et al (JEM-EUSO Coll.), Astroparticle Physics, 10.1016/j.astropartphys.2018.10.008 (2018).

[16] G. Abdellaoui et al (JEM-EUSO Coll.), J. of Instrumentation 1305023 (2018).

[17] G. Abdellaoui et al (JEM-EUSO Coll.), Nucl. Instr. \& Meth. A 866150 (2017).

[18] M. Battisti et al (JEM-EUSO Coll.), Nucl. Instr. \& Meth. A, 10.1016/j.nima.2018.11.002 (2018).

[19] K. Shinozaki et al (JEM-EUSO Coll.), This Conference Proceedings (2018).

[20] P. Klimov et al (TUS Coll.), This Conference Proceedings (2018).

[21] M. Bertaina et al (TUS Coll.), This Conference Proceedings (2018).

[22] A. Belov et al, Advances in Space Research, 10.1016/j.asr.2017.10.044 (2017). 